Notfall Rettungsmed 2010 $13: 508-510$

DOI 10.1007/s10049-010-1348-1

(c) European Resuscitation Council 2010
B. Dirks ${ }^{1} \cdot$ M. Baubin ${ }^{2}$

${ }^{1}$ Sektion Notfallmedizin, Universitätsklinikum Ulm

${ }^{2}$ Universitätsklinik für Anästhesie und Intensivmedizin, Innsbruck,

\title{
Vorwort zu den Leitlinien zur Reanimation 2010 des European Resuscitation Council
}

Notfall + Rettungsmedizin publiziert mit diesem Heft die autorisierte deutschsprachige Übersetzung der aktualisierten Leitlinien zur Reanimation 2010 des European Resuscitation Council (ERC) durch den Deutschen Rat für Wiederbelebung - German Resuscitation Council (GRC), den Österreichischen Rat für Wiederbelebung - Austrian Resuscitation Council (ARC) und den Schweizer Rat für Wiederbelebung - Swiss Resuscitation Council (SRC).

In seinem Editorial „The ILCOR ${ }^{1}$ process for developing guidelines" beschreibt Dr. Jerry Nolan, der Co-Chairman des ILCOR Prozesses 2010, sehr eindringlich, welche umfassende und genaue Auswertung der Literatur, ganz zu schweigen von der immensen Detailarbeit, die Teilnehmer des ILCOR Prozesses geleistet haben, bis der Consensus on Science and Treatment Recommendations (CoSTR) als Konsens der Wissenschaft der Reanimation und als Basis für die Redaktionsgruppen („Writing Groups“) den Reanimationsgesellschaften der Welt zur Verfügung stand.

$\mathrm{Ab}$ April diesen Jahres konnten dann für Europa die „Writing Groups“ des ERC daran gehen, diese wissenschaftliche Basis in aktualisierte Leitlinien für die Bereiche Lebensrettende Basismaßnahmen, Erweiterte Reanimationsmaßnahmen, Elektrotherapie, das akute Koronarsyndrom, Lebensrettende Maßnahmen bei Kindern, Versorgung des Neugeborenen sowie die speziellen Aspekte Ausbildung und Ethik

${ }^{1}$ ILCOR „International Liaison Committee on Resuscitation". umzusetzen. Dies haben zur gleichen Zeit auch die Kolleginnen und Kollegen der anderen Kontinente für ihre Bereiche geleistet, so dass auch für Nordamerika (American Heart Association (AHA), Heart and Stroke Foundation of Canada (HSFC)), Australien und Neuseeland (ANZCOR), Asien, Südafrika und Südamerika eigene Leitlinien erscheinen. ERC, AHA, HSFC und ANZCOR können in ihren Kontinenten auf etwa gleiche notfallmedizinische Systeme und vergleichbare Bildung der Bevölkerung aufbauen. So unterscheiden sich diese Leitlinien nur marginal (wie auch zwischen den Staaten Europas) in ihren auf dem CoSTR beruhenden Leitlinien. Es gibt allerdings immer geringe Unterschiede, die sich aus der unterschiedlichen Struktur, Geographie und Vorgeschichte erklären. Die Unterschiede scheinen uns mit Veröffentlichung der Leitlinien zur Reanimation 2010 weiter zurückgegangen zu sein, man kann sagen: Diese Leitlinien geben die weltweit akzeptierte Sicht wieder, wie Reanimation wirkungsvoll und sicher durchgeführt werden kann.

Obwohl der Kreislaufstillstand beim Menschen nicht immer auf die gleiche Ursache zurückzuführen ist [1] und folglich Ursachenabhängig unterschiedliche Prioritäten für die Reanimation zu setzen wären, geben die Leitlinien für den Laienhelfer (BLS) ein Standardvorgehen vor, das zwar dem größten Teil der Betroffenen gerecht wird (Kreislaufstillstand aus kardialer Erkrankung) aber nicht für alle Ursachen den effektivsten Weg darstellt. Leitlinien beschreiben deshalb immer einen „Korridor“, von dem in Einzelfällen Ursachenbedingt abgewichen werden kann und soll. Die aktuellen Leitlinien bestätigen für die Laienreanimation den 2005 gefundenen Kompromiss: „Jeder Notfallzeuge mit und ohne Reanimationstraining muss einem Mitbürger, der einen Kreislaufstillstand erleidet, durch Herzdruckmassage helfen“. Diese soll mindestens $5 \mathrm{~cm}$ tief und mit einer Frequenz von mindestens $100 / \mathrm{min}$ ohne Unterbrechung fortgeführt werden, bis der Rettungsdienst eintrifft und übernimmt. Ausgebildete Helfer sollen zusätzlich im Verhältnis 30:2 so beatmen, dass sich in ca. 1 sek die Brust deutlich sichtbar hebt (500 - $600 \mathrm{ml}$ Atemhubvolumen).

Um dieses Ziel „Herzdruckmassage durch jeden " zu erreichen, wird in unseren deutschsprachigen Ländern den Leitstellen eine hohe Verantwortung zukommen. Der Leitstellendisponent ist häufig der Schlüssel zu einem neurologisch intakten Überleben des Patienten. Er ist im Stande, durch präzise (Protokoll gebundene) Abfrage der Notfallmeldung den Kreislaufstillstand zu erkennen und dem Notfallzeugen die Unsicherheit zu nehmen, ob es sich wirklich um einen Kreislaufstillstand handelt. Auch wenn die Leitlinien den Laien ausdrücklich vor der Verkennung der agonalen Schnappatmung warnen, wird ihm doch erst der professionelle Disponent die Sicherheit geben: Jetzt muss ICH es tun. Studien aus dem deutschen Raum [2] weisen deutlich darauf hin, dass Unsicherheit DER Grund für Untätigkeit ist. Zweitens ist die Herzdruckmassage gut telefonisch zu erklären. Der Leitstellendisponent ist im Stande zu erfolgreicher Re animation durch Herzdruckmassage anzuleiten. Sicherlich war es vor 2005 


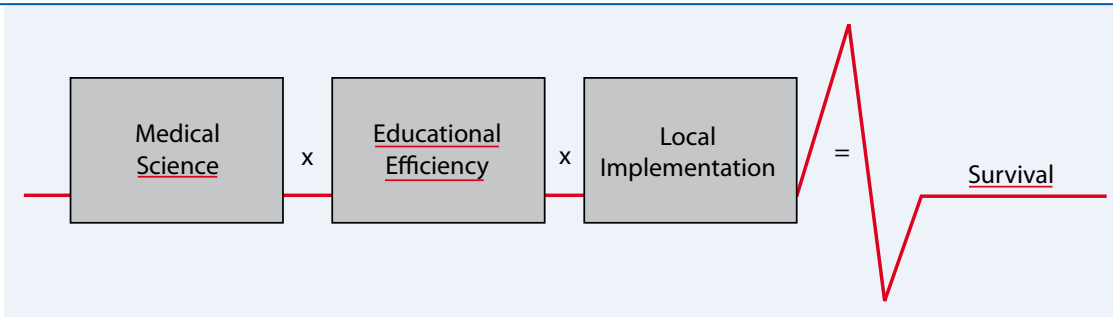

Abb. $1 \Delta$ Die Überlebensformel (modifiziert nach [5])

schwierig, einen nie ausgebildeten Laien telefonisch zur Beatmung anzuleiten. Aber seit 2005 die Maxime galt: „Wer nicht beatmen kann oder will, soll drücken statt nichts zu tun“, war der Weg zur „Telefonreanimation“ frei. Konsequenterweise legt der ERC 2010 einen Schwerpunkt auf die „Telefonreanimation“. In den deutschsprachigen Ländern sollte diese Strategie auch dadurch erfolgreich sein, dass ja ein hoher Prozentsatz der Bevölkerung durch die vorgeschriebenen „Erste Hilfe Kurse“ (z.B. zum Führerschein) verschüttete oder veraltete Vorkenntnisse aufweisen, die in der konkreten Situation aktiviert werden können. Die Tatsache, dass praktisch jeder heute an jedem Ort, zu Hause und unterwegs ein Telefon bei sich hat, erleichtert dem Disponenten die Anleitung.

Warum ist dies so wichtig? Jedes Jahr sterben in unseren Ländern 100 ooo Menschen, die nicht erfolgreich reanimiert werden konnten, nur in 10-15\% versucht ein Notfallzeuge Basisreanimation zu leisten. Die wenigsten Laien machen sich klar, dass der Kreislaufstillstand ein Notfall ist, dessen zeitliche Dynamik den leistungsfähigsten Rettungs- und Notarztdienst überfordert. Das Gehirn stirbt - ohne Herzdruckmassage - nach 5 min, Hilfe naht (im Durchschnitt) in Deutschland, Österreich und der Schweiz günstigstenfalls nach 10 min (Rettungsmitteleinsatzzeit + Meldung und Disposition). Diese zeitliche Lücke müssen wir durch Laienreanimation schließen. Voranbringen können uns:

- Das Problem und seine Größe be-

kannt und die Menschen betroffen zu machen, damit jedem Bürger bewusst ist, dass es auf ihn ankommt.

- Die Telefonreanimation flächendeckend einzuführen und sicherzustellen.

- Das Wissen, wie man richtig wiederbelebt als Bildung jedes Bürgers in die Schulen zu bringen.
Aber es geht beim Leitlinien Update 2010 nicht nur um Fortschritt bei den Notfallzeugen. Auch professionelle Helfer können ihre Maßnahmen weiter verbessern und damit zu einem neurologisch intakten Überleben der Patienten beitragen.

In Kliniken ist das frühe Erkennen und Beseitigen von Störungen der Vitalfunktionen, die zu einem Kreislaufstillstand führen können, nicht selbstverständlich obgleich unsere Patienten sich eigentlich darauf verlassen. Die ALS Leitlinien des ERC gehen auf dieses Problem intensiv ein. Die Botschaft ist: Es darf in der Klinik gar nicht erst zum Kreislaufstillstand kommen, wenn aber doch, dann muss qualifizierte Hilfe in 3 min verfügbar sein.

Die Erkenntnis, das schon 10 sek Unterbrechung der Thoraxkompression die Chancen verschlechtern, führte auch zu Änderungen der erweiterten Reanimationsmaßnahmen (ALS). Revolutionär wird manchem vorkommen, dass wir jetzt empfehlen, während laufender Herzdruckmassage den Defibrillator zu laden und in Kauf nehmen, dass der Sicherheitsabstand zwischen Drücken und Strom buchstäblich kleiner wird - aber er reicht! Mit dieser Maßnahme soll die Defibrillation die Thoraxkompression nur 5 sek unterbrechen. Auch beim Atemwegsmanagement wird mancher heilige Kühe geschlachtet sehen. Professionelle Reanimation bedeutet nicht zwangsläufig, dass der Patient intubiert wird, die Intubation als Atemwegssicherung wird nur empfohlen, wenn sie mit nur 10 sek Unterbrechung der Thoraxkompression möglich ist, sonst sollen Alternativen verwendet werden. Grund ist, dass der denkbare Vorteil eines optimal gesicherten Atemweges schnell durch die Unterbrechung der Thorax kompression zunichte gemacht wird.

Am empfohlenen Reanimationsablauf von Kindern ändert sich wenig. Aber der grundsätzliche Umgang mit dem Pro- blem, dass Kinderreanimationen - Gott sei Dank - selten sind und der Notfallzeuge dann sicher nicht mehr irgendwelche speziellen Maßnahmen für Kinder erinnert, ist weiter pragmatischer geworden. Das Prinzip ist: Wer nicht besondere Verantwortung für Kinder trägt (und sich deshalb auskennen muss), soll Kinder reanimieren wie Erwachsene.

Reife Neugeborene mit peripartaler hypoxischer bzw. ischämischer Enzephalopathie sollen ebenso wie Erwachsene nach Reanimation gekühlt werden.

Leider bleibt die Verbreitung der therapeutischen Hypothermie nach ROSC (Return of Spontaneous Circulation) weit hinter dem zurück, was einer so erfolgreichen Methode zusteht. Die Leitlinien machen noch einmal stark Werbung, jede Klinik muss das Verfahren in ihre Standards einführen. Es gibt keine andere einzelne Maßnahme, die vergleichbar viel Leid verhindert.

Die wahrscheinlich häufigste und wichtigste „peri arrest“ Erkrankung ist das Akute Koronarssyndrom (ACS). Deshalb nimmt der ERC das ACS traditionell in die Leitlinien auf. Zwei Aussagen fallen auch dem Nicht Kardiologen sofort auf:

Die Reperfusionsstrategie ist stärker differenziert als in den Leitlinien der European Society of Cardiology (ESC). Basierend auf der Auswertung des amerikanischen Myokardinfarkt Registers wird die Wahl der Reperfusionsstrategie von Infarktlokalisation, Alter und Symptomdauer abhängig gemacht. Damit wird neben der bevorzugten und im Verlauf der Versorgung auch immer empfohlenen perkutanen Koronarintervention (PCI) zunächst die präklinische Lyse bei einem Teil der Patienten als Reperfusionsstrategie eingesetzt werden müssen (maximaler Zeitverlust zwischen Start der Fibirnolyse und erster Balloninflation 45-180 min). In allen Fällen soll der Patient dann aber in eine Katheterklinik eingewiesen werden.

Zweitens und eine kleine Revolution: Patienten mit ACS, ebenso wie Patienten nach Reanimation, soll nicht mehr unkritisch maximal Sauerstoff angeboten werden. Die Studien, die bei zu hohen Sauerstoffpartialdruck ein verschlechtertes Outcome gefunden haben sind zwar gar nicht neu, sie wurden aber erst jetzt beachtet. Deshalb gilt bei ACS ohne wei- 
tere Probleme und nach Reanimation eine pulsoximetrisch gemessene $\mathrm{SaO}_{2}$ von 94-98\% als ideal.

Alles in allem dürfen wir konstatieren: Die Leitlinien sind weiter einfacher geworden, Brüche zu 2005 gibt es nicht, beides wird die Vermittlung erleichtern.

Wir sollten nie die „Überlebensformel“ [3] aus den Augen verlieren: Überleben ist: Wissenschaft $x$ Ausbildung $x$ Implementierung (• Abb. 1)!

So wird es darauf ankommen, diese Leitlinien Laien und professionellen Helfern zu vermitteln und die zugrunde liegenden erfolgreichen Konzepte zu implementieren an der Spitze die Prinzipien , Jeder kann reanimieren " und „Jeder Patient nach ROSC verdient gekühlt zu werden“.

Ihre

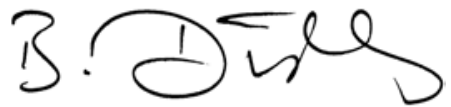

Burkhard Dirks

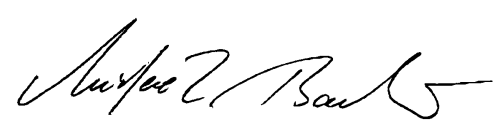

Michael Baubin

\section{Korrespondenzadresse}

Dr. rer. nat. Dr. med. Burkhard Dirks, Sektion Notfallmedizin, Universitätsklinikum Ulm, Prittwitzstr. 43, 89075 Ulm, burkhard.dirks@uni-ulm.de

Interessenkonflikt. Der korrespondierende Autor gibt an, das kein Interessenkonflikt besteht.

\section{Literatur}

1. Pell JP, Sirel JM, Marsden AK et al (2003) Presentation, management, and outcome of out of hospital cardiopulmonary arrest: comparison by underlying aetiology. Heart 89:839-42.

2. Breckwoldt J, Schloesser S, Arntz HR (2009) Perceptions of collapse and assessment of cardiac arrest by bystanders of out-of-hospital cardiac arrest - OOHCA. Resuscitation 80: 108-1113

3. Castrén M, Karlsten R, Lippert F et al (2008) The Emergency Medical Dispatch expert group at the Utstein Consensus Symposium 2005 Recommended guidelines for reporting on emergency medical dispatch when conducting research in emergency medicine - the utstein style. Resuscitation 79:193-197

\section{ㅇ 2010 European Resuscitation Council (ERC)}

Die Beiträge dieser Ausgabe sind durch das Copyright des ERC geschützt und für ihre Nutzung gelten folgenden Bedingungen:

\section{Fotokopieren}

Einzelne Fotokopien von einzelnen Artikeln dürfen für den persönlichen Gebrauch entsprechend der nationalen CopyrightGesetze angefertigt werden. Für alle weiteren Arten des Fotokopierens, d.h. mehrfaches oder systematisches Kopieren, Kopieren für Anzeigen oder sonstige Werbung, Weiterverkauf und alle sonstigen Arten der Dokumentenlieferung muss eine kostenpflichtige Zustimmung des ERC eingeholt werden.

\section{Abgeleitete/sekundäre Arbeiten}

Abonnenten dürfen Inhaltsverzeichnisse oder Listen von Artikeln einschl. Abstracts für den internen Gebrauch in ihren Institutionen anfertigen. Für den Weiterverkauf oder Verteilung außerhalb der Institution ist die Erlaubnis des ERC einzuholen. Auch für alle anderen sekundären Nutzungen, einschl. Sammlungen und Übersetzungen, ist die Erlaubnis des ERC nötig.

\section{Elektronische Sicherung oder Nutzung}

Für die elektronische Sicherung oder Nutzung, ganz oder teilweise, ist die Erlaubnis des ERC einzuholen.

Ohne die vorherige schriftliche Erlaubnis des ERC darf kein Teil dieser Publikation - abgesehen von den o.g. Bedingungen - reproduziert, weiter gegeben oder in einer Datenbank gespeichert werden, sei es elektronisch, mechanisch, durch Fotokopieren, durch Aufzeichnung oder sonstige Mittel.

\section{Hinweis}

ERC übernimmt keine Verantwortung für Personen- und/oder Sachschäden, die aus Produkthaftung oder Fahrlässigkeit oder durch irgendwelche Anwendung oder Handlung jedweder Methoden, Produkte, Anweisungen oder Ideen, die in diesen Publikationen enthalten sind, entstehen. Aufgrund des raschen Fortschrittes der medizinischen Wissenschaft sollte insbesondere eine unabhängige Überprüfung der Diagnose und Dosierung von Medikamenten vorgenommen werden.

\section{Deutsche Fassung}

Die Übersetzung der deutschen Version der „European Resuscitation Council Guidelines for Resuscitation 2010" erfolgte durch die deutschen, österreichischen und schweizerischen Mitglieder des ERC.

Erstellt für das European Resuscitation Council durch H.-R. Arntz, J. Bahr, M. A. Baubin, S. Braunecker, G. Burda, B. Dirks, C. Eich, H. W. Gervais, P. Goldschmidt, J.-T. Gräsner, R. Greif, U. Klemmer, U. Kreimeier, U. Kreth, S. Osche, S. Russo, M. Sasse, J. C. Schwindt und V. Wenzel.

Die deutsche Übersetzung wurde autorisiert durch B. Dirks, Ulm, und M. Baubin, Innsbruck (Vertreter für Deutschland und Österreich im Executive Committee des ERC).

Die Rechte an der deutschen Fassung werden durch den Springer-Verlag wahrgenommen. Anfragen zu einer Veröffentlichung und der sonstigen Verbreitung durch Dritte müssen über den Springer Verlag an die deutschen und österreichischen Vertreter im Executive Committee des ERC gerichtet werden. 